\title{
THE CHARACTERISTICS OF ACETABULAR CUPS WORN IN THE HUMAN BODY
}

\author{
JUDITH M. DOWLING, J. R. ATKINSON, D. DOWSON, JOHN CHARNLEY \\ From the University of Leeds and Wrightington Hospital, Wigan
}

\begin{abstract}
In laboratory tests, the ultra-high molecular weight polyethylene used for the acetabular cups of Charnley hip prostheses has a very low wear rate against steel. In the body radiographic measurements indicate that the polyethylene wears more rapidly. In order to investigate this higher wear rate, the sockets of acetabular cups removed at post-mortem have been examined using optical and electron microscopy. It has been shown that a socket wears predominantly on its superior part and that this is a direct consequence of the orientation of the cup in the body and the direction of loading of the hip. In the worn region the femoral head in effect bores out a new socket for itself, a process which is visible with the naked eye after approximately eight years. Electron microscopy shows that the predominant wear mechanism is adhesion, but after about eight years the appearance of surface cracks suggests that surface fatigue is taking place in addition to this. Laboratory wear tests have shown that pure surface fatigue is not sufficient to account for the high clinical wear rate. Other deformation processes are suggested and discussed with regard to the higher clinical wear rate.
\end{abstract}

This report describes the appearance of the load-bearing surfaces of the acetabular cups of Charnley total hip replacements (THR) after service in the human body (Fig. 1). This hip prosthesis has been used for about fifteen years, mainly in elderly patients suffering from osteoarthritis. It consists of an acetabular cup and a femoral prosthesis. The cup is machined from a block of isotropic ultra-high molecular weight polyethylene (UHMWPE), a material which has good mechanical properties, a low friction coefficient against steel, good wear resistance, is non-toxic and also has non-toxic wear debris (Atkinson 1975). The femoral prosthesis is made of stainless steel (316L) which is thermomechanically treated to achieve the desired microstructure and optimal mechanical properties, and then machined to the required shape and polished. This material is strong and tough, has a high resistance to chemical attack and, because it is harder than UHMWPE, has a negligible wear rate when rubbed against this plastic.

At present there is relatively little known about the deformation and wear behaviour of UHMWPE cups in the human body. The clinical "wear" of UHMWPE cups has been assessed from patients' radiographs and the mean "wear" rate found to be about 0.1 millimetre per annum (Charnley and Cupic 1973). However, accelerated laboratory wear tests (Atkinson, Brown and
Dowson 1978) on UHMWPE sliding on stainless steel in dry conditions, using a load equivalent to body weight and a sliding speed near to that in the human hip, give a mean wear rate of about $3.0 \times 10^{-7} \mathrm{~mm}^{3} \mathrm{~N}^{-1} \mathrm{~m}^{-1}$, equi-

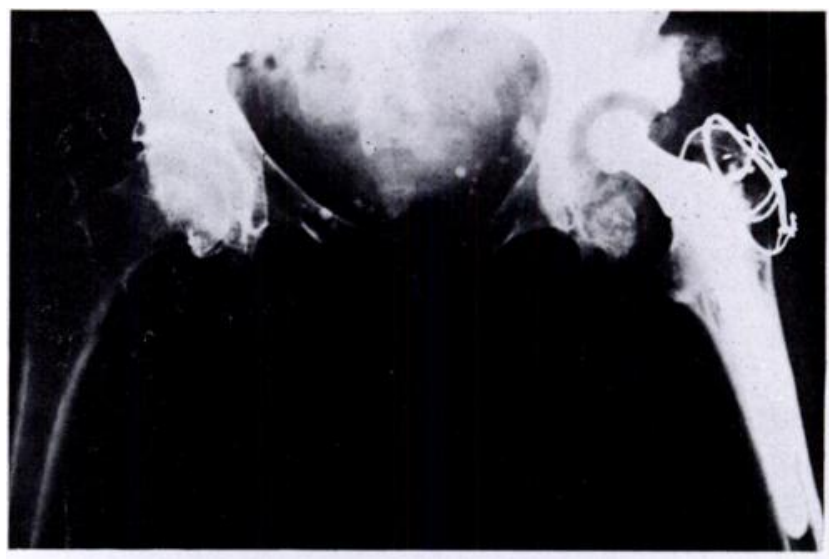

Fig. 1

Charnley total hip replacement in vivo.

valent to about 0.02 millimetre per annum. These laboratory tests show that initially the plastic wears adhesively at a rate of $2.3 \times 10^{-7} \mathrm{~mm}^{3} \mathrm{~N}^{-1} \mathrm{~m}^{-1}$. (Adhesive wear often involves the transfer of material from one

Judith M. Dowling, M.A., D.Phil., M.I.M., Departments of Metallurgy and Mechanical Engineering

J. R. Atkinson, M.Sc., D.Phil., Department of Metallurgy

D. Dowson, B.Sc., Ph.D., D.Sc., C.Eng., F.I.Mech.E., Fellow A.S.M.E., A.S.L.E., $\}$ Leeds LS2 9JT, England.

Department of Mechanical Engineering

Professor Sir John Charnley, C.B.E., F.R.S., F.R.C.S., Centre for Hip Surgery, Wrightington Hospital, Wigan, Lancashire, England.

Requests for reprints should be sent to Dr J. R. Atkinson. 
surface to another, during relative motion, as a consequence of adhesive forces.) After long sliding times the wear rate increases to $3.6 \times 10^{-7} \mathrm{~mm}^{3} \mathrm{~N}^{-1} \mathrm{~m}^{-1}$. The UHMWPE continues to wear adhesively, but there are signs of surface fatigue as well and it is believed that this is the cause of the increased wear rate. This surface fatigue is characterised by the appearance of small cracks (about 2 micrometres long) on the polymer surface and spall marks, where the cracks have propagated under the surface leading to the detachment of flakes of polymer. Surface fatigue occurs when both surfaces are relatively smooth and the stress varies cyclically. In terms of the sliding conditions in the Charnley THR, the onset of surface fatigue has been predicted (Atkinson et al. 1978) after eight to nine years of service in the human body and preliminary investigations support this hypothesis (Brown et al. 1976). system is run dry or, if wet, using a stable fluid such as water; the temperature is at the ambient level. Such simplicity is not found in the natural hip or in the THR, where the following factors must be considered: the force on a hip is not static and in walking, for example, the force varies between zero and about four times body weight (Paul 1976); the movement of the femoral head in its socket is very complicated, for it can rotate in three different planes and superimposed on this are rolling and sliding (MacConaill 1966); the sliding speed varies from zero to probably $100 \mathrm{~mm} \mathrm{~s}^{-1}$ (Dowson 1966-67); the THR is almost certainly not a dry bearing and the fluids present are probably not chemically inert; the temperature of the hip will be about body temperature.

From the above comments it is clear that laboratory tests on their own are not sufficient to predict the long-term wear and deformation of the UHMWPE cup

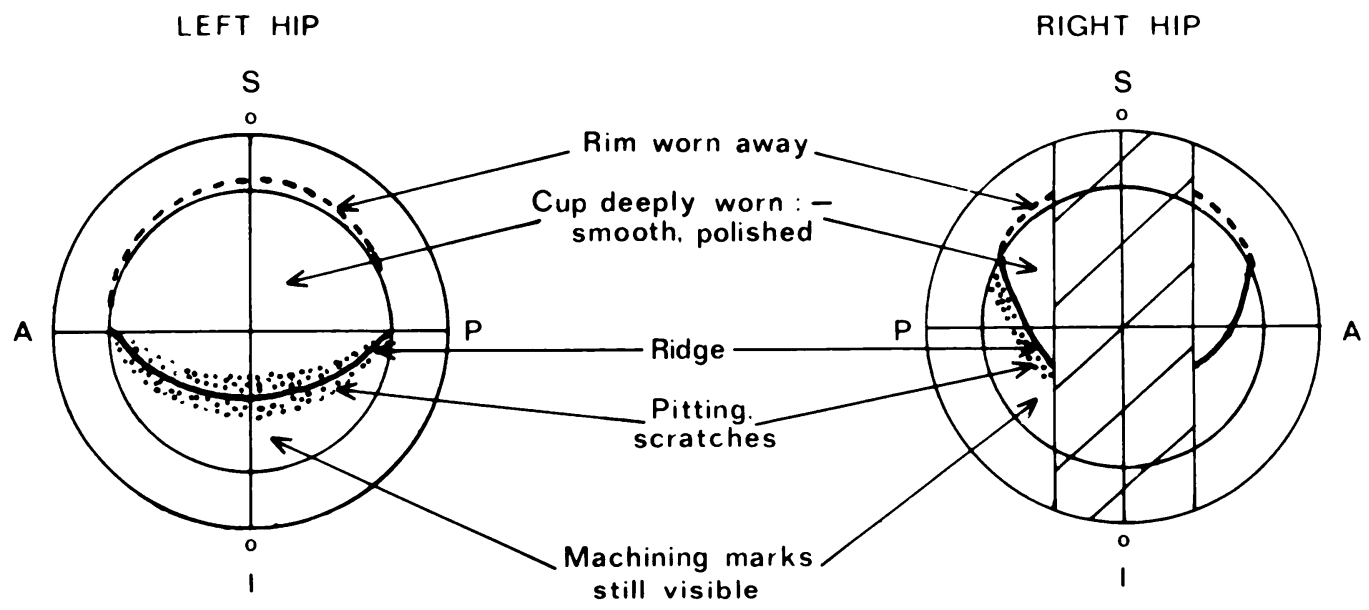

Fig. 2

Appearance of two acetabular cups which have been worn in the human body (Patient HB3). S, superior; P. posterior; I, inferior; $A$. anterior. (Not to scale.) The clinical details of this patient were: age of left hip 9 years 9 months, and of right hip 9 years 11 months; age at time of operation 74 years; weight 7 stone 4 pounds; activity, light housework; gait normal; walking ability 2 to 3 miles.

The above laboratory tests were run dry whereas fluid is found in the joint capsule of THR (Charnley 1978). Laboratory tests on UHMWPE sliding against 316L stainless steel have shown that, when the sliding system is lubricated, the wear rate of the polymer is less than in dry sliding (Duff-Barclay and Spillman 196667). Therefore, the higher clinical "wear" rate suggests that other deformation processes, as well as true wear, take place in the human body. (The terms wear and worn as used here indicate true wear; "wear" and "worn" are used for a combination of true wear and other processes.)

When comparing laboratory wear tests with clinical "wear" rates, it must be remembered that laboratory sliding conditions are often very simple. The applied load is usually static; the sliding motion is linear or reciprocating; a constant sliding speed is used; the in the human body. Some "in situ" investigations are required. The most convenient method is to examine worn acetabular cups after removal post mortem or at revision operations.

\section{EXPERIMENTAL METHODS}

The acetabular cups examined so far were all removed post mortem and had been stored in formalin. Several had been cut in half for other research and in some cases only half a cup was available.

Initially all the cup surfaces were examined with the naked eye and a hand lens. Visible surface features were recorded using a schematic mapping system. An example of this is shown in Figure 2; the spotted areas denote parts of the cup that were missing due to sectioning.

Transmission electron microscopy is necessary to study the microscopic surface features and for this a two-stage replication technique was used. A square (about $2 \times 2$ millimetres) of thin cellulose acetate film (Bex film) was softened in acetone and lightly pressed onto the surface. When dry, the film was lifted off, turned upside down and "shadowed" by evaporating a heavy metal at an 


\section{LEFT HIP}
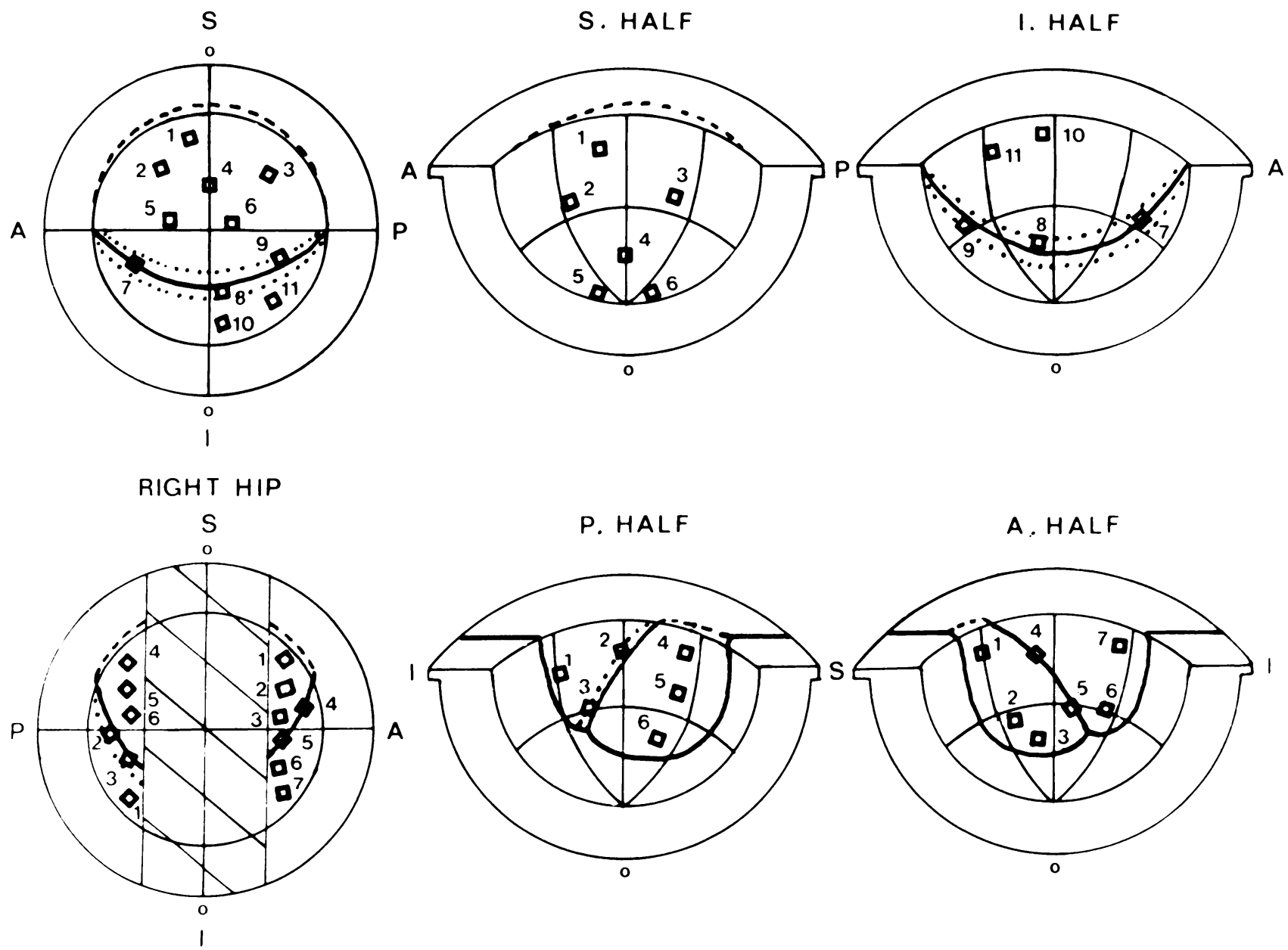

Fig. 3

Approximate positions of the plastic replicas taken from the surfaces of the worn acetabular cups shown in Figure 2.

angle onto the replica surface to accentuate the fine-scale topography. A thin film of carbon was then evaporated onto the replica in a planar fashion to serve as a support medium. The cellulose acetate film was dissolved away as it is not stable in the electron beam of the microscope. The resulting shadowed carbon replica was then examined in a conventional transmission electron microscope, a Philips 300, operated at $100 \mathrm{kV}$.

The advantages of the above replication method are that the plastic film is a faithful copy of the specimen surface, even for features of the order of 0.1 micrometre across, and the specimen surface is not destroyed so that the same surface can be replicated more than once.

In order to correlate features seen on the replicas with those observed optically, the positions of the replicas on each cup were recorded using the mapping system shown in Figure 3.

\section{RESULTS}

To date, twenty-one acetabular cups from seventeen patients have been examined optically and of these fourteen have been examined using transmission electron microscopy as well. The age of these cups ranges from one to fourteen years. For simplicity, this report concentrates mainly on the acetabular cups from one patient (HB3) whose record sheets are shown in Figures 2 and 3. This patient had two arthroplasties performed when she was seventy-four years old and was quite active in the ten years after the operations.

Optical microscopy. The visible surface features of this patient's acetabular cups are quite representative of other cups examined. On all cups there are two main "wear" areas. On the superior half of the socket, the surface is smooth and well polished (Fig. 2) - this is referred to as the "high wear" area. The inferior part of the socket-the "low wear" area-resembles the surface of an unused cup: the parallel lines produced by machining are visible, but these have been worn down by the femoral head, leaving only the valleys.

The shapes of the high and low wear areas reflect the loading geometry of the hip. The acetabular cup is inserted at an angle of approximately 45 degrees to the vertical (Fig. 1). The force on the head and neck of the femur (joint force $J$ ) operates in a direction inclined at 10 to 15 degrees to the centre line of the body (Paul 1976). When standing or walking, this joint force is the resultant of the body weight acting downwards and the force exerted by the abductor muscles. By considering the line of action of the joint force and the orientation of 
the acetabular cup (Fig. 4), the shape of the contact area between the femoral head and the cup socket can be determined qualitatively. From Figure 4 it is seen that the maximum area through which stress can be transmitted to the socket is determined by the crosssectional area of the femoral head perpendicular to the direction of the joint force-the circular plane denoted by the line $X Y$ in Figure 4 . When a load is applied to the hip, for example during walking, contact will occur over the hemispherical area above the plane XY. The area of

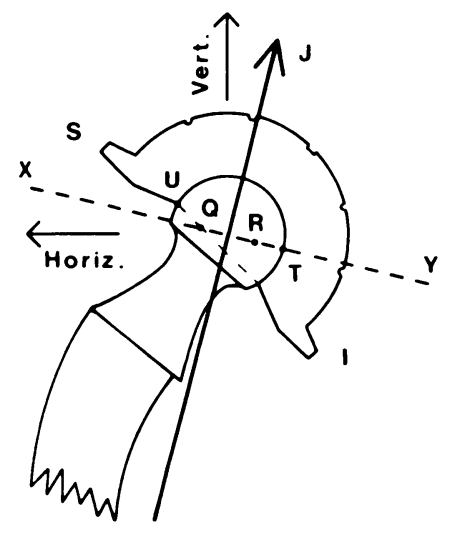

Fig. 4

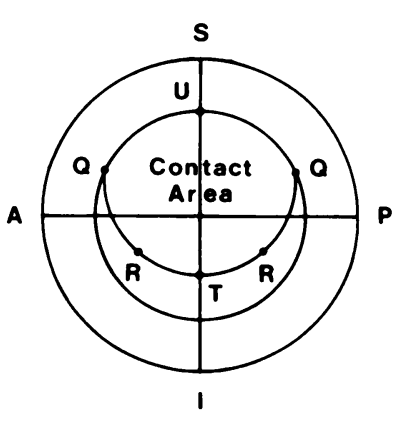

Fig. 5
Orientation of the Charnley hip joint and joint force, $J$, in relation to the observed contact area on the acetabular cup. Figure 4-Loading geometry of the hip (see text). Figure 5-Main contact area on acetabular cup obtained by plotting points $U, Q, R$ and $T$ seen in Figure 4 .

contact is then bounded by the line where the circular plane $X Y$ intersects the socket surface. If $U$ is a point on the superior part of the rim, and $Q, R$ and $T$ are points where $X Y$ intersects on the socket surface, and these are plotted on a schematic plan of the socket (Fig. 5), then the contact area obtained is similar to that seen on sockets worn in the human body.

The shape of the contact area also explains other phenomena. In cups "worn" for a short time, for example one year, the high wear area is smaller than that seen in Figure 2. Some time later, after perhaps two or three years, the high wear area seems to reach a maximum: there is little difference in size between the high wear area of a five-year-old cup and that of a ten-year-old cup. The reason for this is that initially there is a "wearing in" period where surface irregularities, which prevent complete surface contact, are removed. This process is apparently completed rapidly, after which the contact area is that predicted from theory.

Another feature, usually associated with heavily worn cups, is a sharp ridge between the high and low wear areas. An example of this is seen in Figures 2 and 3, on the sections of the right cup. This ridge appears to be a direct result of the loading, and hence the preferential "wear", on the superior half of the cup. With time, the socket in the high wear area is "worn" away and deepens in this region-the femoral head effectively bores out a new socket for itself (Fig. 6). A typical depth for this new socket is 1 to 2 millimetres after about ten years. A ridge is not observed in patients of low activity or in cups worn for less than seven to eight years (Table I).

Pitting and flaking is sometimes seen between the high and low wear areas (Fig. 2). Again this is normally associated with heavily worn sockets. Finally, rim wear is a common feature (Fig. 2). This must result from impingement of the femoral prosthesis during locomotion. Rim wear is restricted to the superior part of the socket, in the high wear area.

Transmission electron microscopy. In the high wear area, adhesive wear features are seen. These can look like fans (Fig. 7) or long thin smears (Fig. 8). In both cases these are produced by parts of the polymer surface adhering to the metal femoral head which pulls and smears out the polymer. Normally, due to the complicated sliding motion of the femoral head, these features follow no specific direction.

In the low wear area the surface is quite different. Worn machining marks are seen, together with ploughing or scratch marks (Fig. 9). This is indicative of abrasive wear. Long cracks, between 10 and 30 micrometres in length, and wrinkles are also seen and these are usually found near the boundary of high and low wear (Fig. 10). The origin of these cracks and wrinkles is uncertain, but possible mechanisms are discussed later.

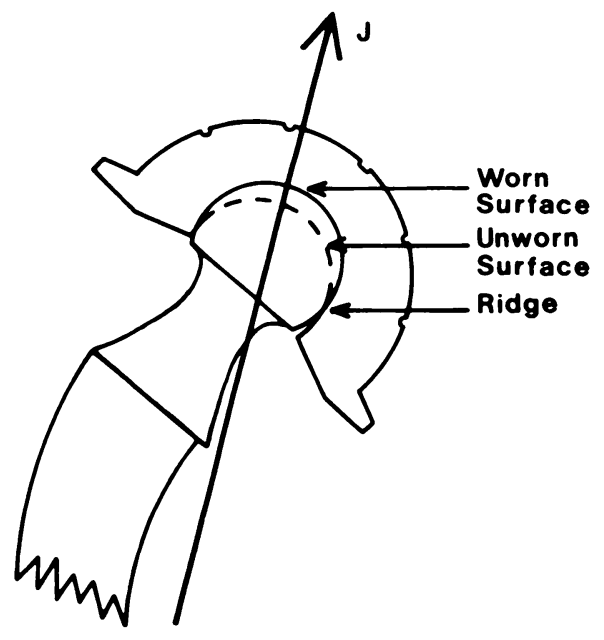

Fig. 6

Boring out of a new socket by the femoral head in the high wear area.

In the border area around the ridge, there is evidence of both adhesive wear and long cracks. It appears that the cracks predominate on the underside of the ridge and range in size from 5 to 20 micrometres (Fig. 11). The adhesive wear is probably confined to the upper side of the ridge. Where pitting and flaking is observed optically, there is evidence of spalling in the corresponding replicas, as seen in Figure 12. 


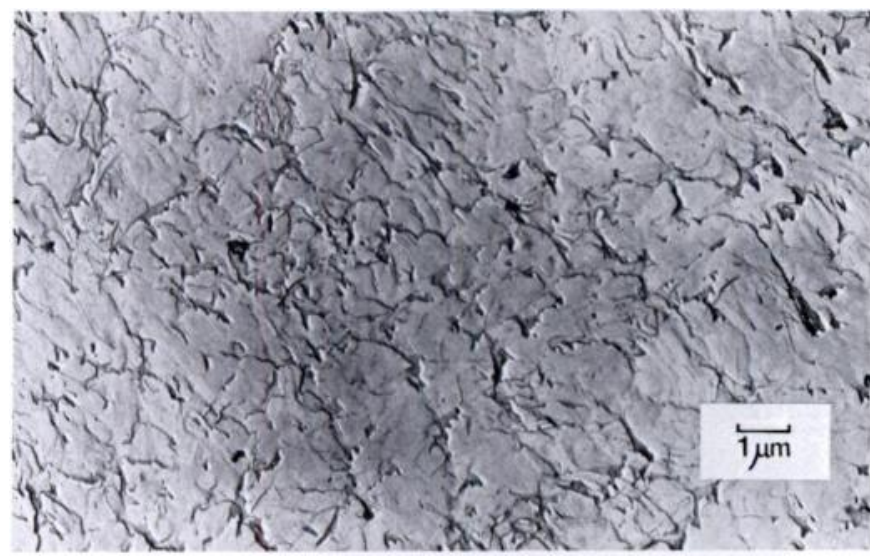

Fig. 7

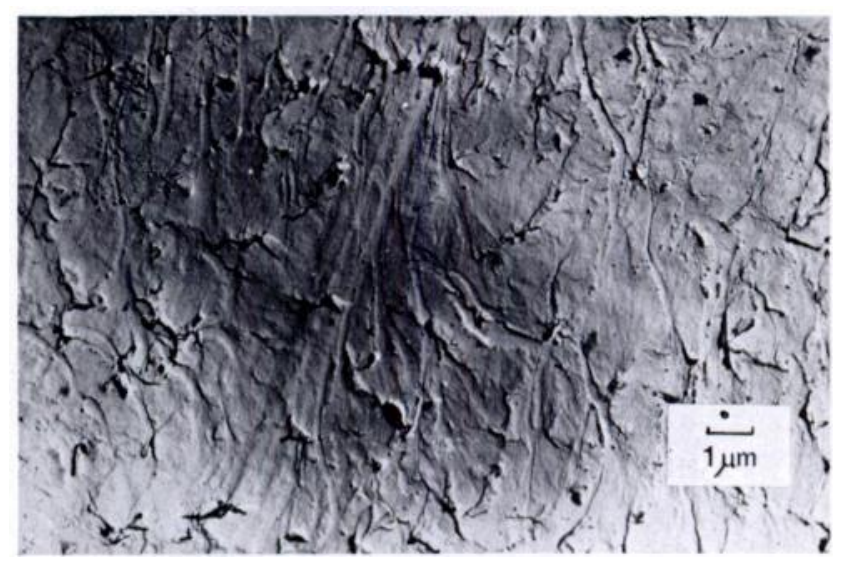

Fig. 8

High wear area on the left acetabular cup from Patient HB3. Figure 7-Adhesive wear, fan type (Replica 4). Figure 8-Adhesive wear, pulls (Replica 2).

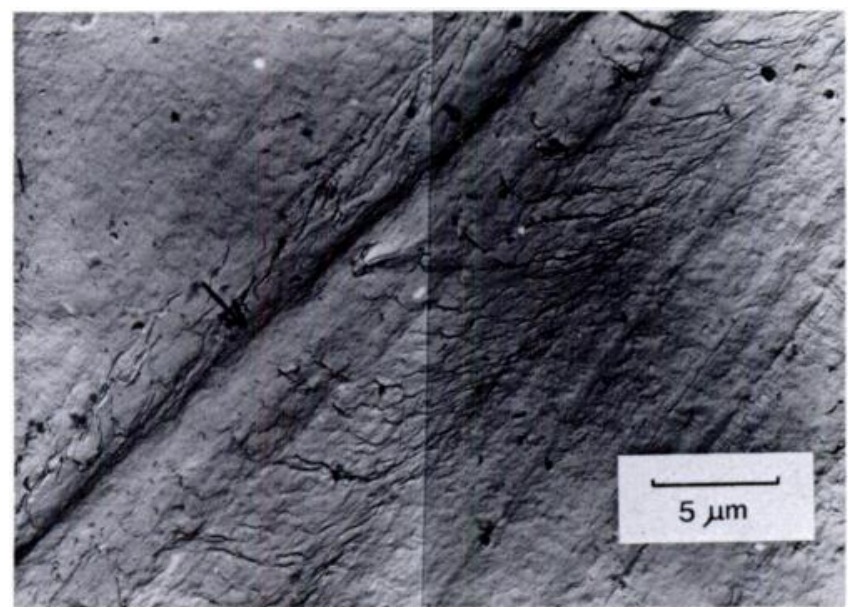

Fig. 9

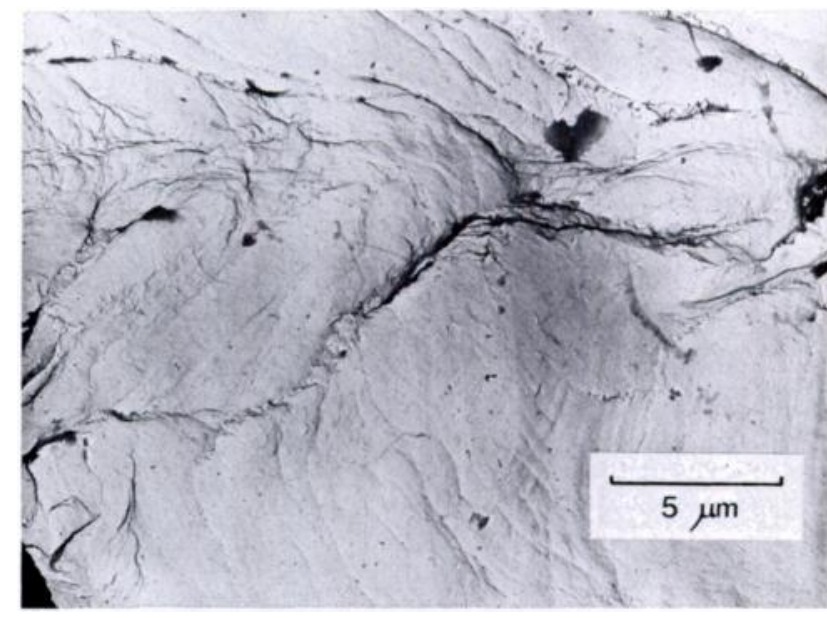

Fig. 10

Low wear areas on acetabular cups. Figure 9-Worn machining marks with scratch mark (arrowed). (Patient HB4.) Figure 10-Cracks and wrinkles. (Patient HB5.)

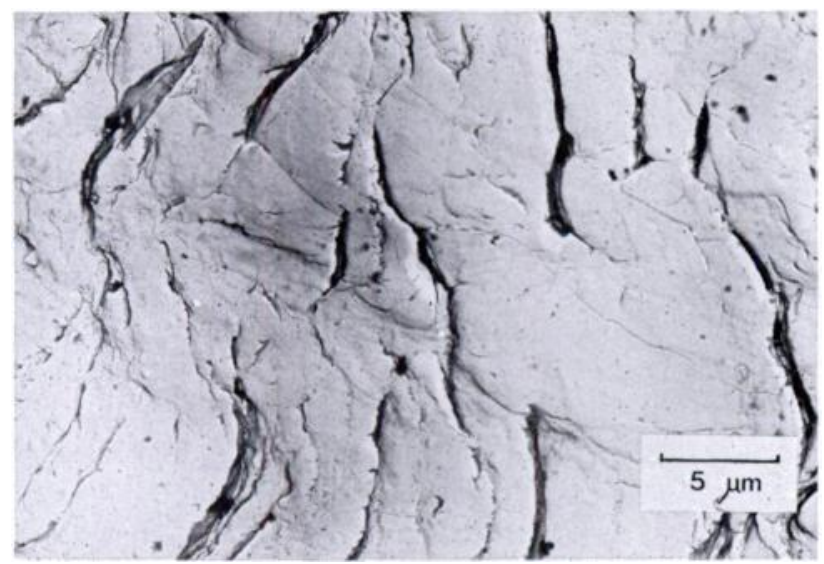

Fig. 11

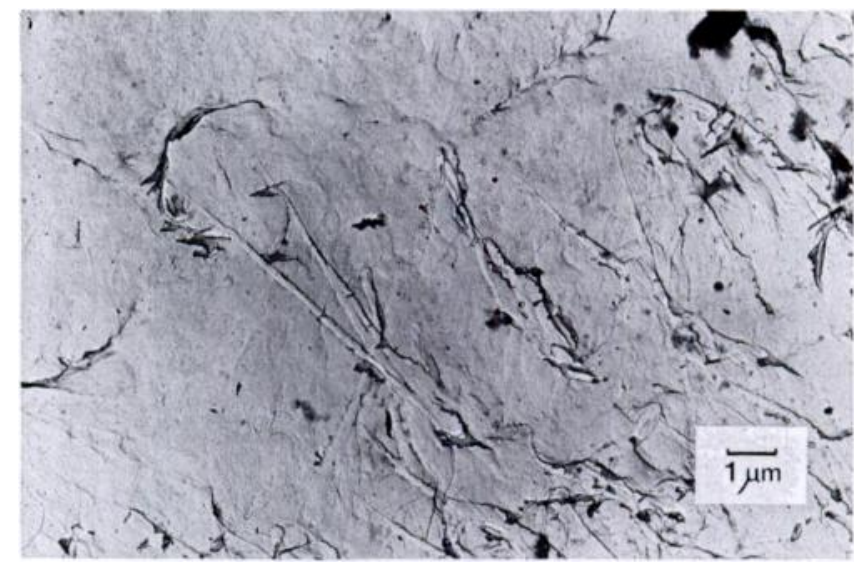

Fig. 12

Border area on the right acetabular cup from Patient HB3. Figure 11-Cracks (Replica 3, posterior half). Figure 12-Adhesive pulls at edge of surface pit (Replica 4, anterior half). 
Table I. The principal wear features in relation to the service age of the prosthesis and the activity of the patient

\begin{tabular}{|c|c|c|c|c|c|c|}
\hline Hip & $\begin{array}{c}\text { Age at operation } \\
\text { (years) }\end{array}$ & $\begin{array}{c}\text { Weight } \\
\text { (kilograms) }\end{array}$ & Service age of hip & Ridge & $\begin{array}{l}\text { Parallel } \\
\text { ripples }\end{array}$ & Fatigue cracks \\
\hline \multicolumn{7}{|l|}{ Grade $C$} \\
\hline $6 \mathrm{HB}$ left & 70 & 92.3 & 1 year 2 months & No & No & No \\
\hline 10HB right & 71 & 44.6 & 2 years 5 months & No & No & No \\
\hline $9 \mathrm{HB}$ left & 75 & 60.9 & 5 years & No & Some & No \\
\hline $1 \mathrm{HB}$ left & 64 & 65.5 & 5 years 2 months & Not distinct & No & No \\
\hline $10 \mathrm{HB}$ left & 67 & 44.6 & 6 years 3 months & No & Some & No \\
\hline $2 \mathrm{HB}$ right & 65 & 61.4 & 7 years 1 month & Not determinable ${ }^{\dagger}$ & * & $*$ \\
\hline $2 \mathrm{HB}$ left & 65 & 61.4 & 7 years 5 months & Yes & No & No \\
\hline $4 \mathrm{HB}$ right & 73 & 67.5 & 8 years & Not distinct & Yes & No, but spalls \\
\hline $8 \mathrm{HB}$ left & 60 & 50.2 & 8 years 11 months & Slight & Some & No \\
\hline $8 \mathrm{HB}$ right & 60 & 50.2 & 9 years 1 month & No & Yes & No \\
\hline $11 \mathrm{HB}$ right & 76 & 66.8 & 10 years 1 month & No & Some & No \\
\hline \multicolumn{7}{|l|}{ Grade B } \\
\hline $12 \mathrm{HB}$ left & 74 & 65.5 & 9 years 8 months & No & $*$ & $*$ \\
\hline $14 \mathrm{HB}$ left & 74 & 50.1 & 9 years 8 months & No & $*$ & * \\
\hline $3 \mathrm{HB}$ left & 75 & 46.4 & 9 years 9 months & Yes & No & Yes \\
\hline 3HB right & 75 & 46.4 & 9 years 11 months & Yes & No & Yes \\
\hline \multicolumn{7}{|l|}{ Grade $A$} \\
\hline 5HB left & 70 & 73.2 & 3 years 8 months & Not distinct & No & No \\
\hline $13 \mathrm{HB}$ left & 61 & 81.8 & 7 years 8 months & Yes & * & $*$ \\
\hline $17 \mathrm{HB}$ right & 67 & - & 8 years 5 months & Yes & $*$ & $*$ \\
\hline $16 \mathrm{HB}$ left & 59 & - & 8 years 6 months & Yes & $*$ & $*$ \\
\hline 7HB left & 77 & 59.1 & 11 years 5 months & Yes & Yes & No, but spalls \\
\hline $15 \mathrm{HB}$ right & 71 & 75.6 & 13 years 3 months & Yes & $*$ & $*$ \\
\hline
\end{tabular}

Hip grades: A-Unilateral hip condition; all other systems related to walking are normal.

B-Bilateral hip condition; all other systems related to walking are normal.

C-There are other factors (apart from hip or hips) which affect walking; for example gross obesity, rheumatoid arthritis of the knee.

* Transmission electron microscopy examination not yet carried out.

† Only "low wear" section available.

The above topography is typical of many acetabular cups, but additional and sometimes very different features are observed in other sockets.

In the high wear area of Patient HB3, fine cracks about 2 micrometres long have been seen (Fig. 13). These are thought to result from surface fatigue and are similar to fatigue cracks observed in accelerated laboratory wear tests (Brown et al. 1976). These fine cracks are quite difficult to locate and can be confused with the edges of adhesive smears and pulls. Fatigue cracks have not been identified with any confidence in the high wear areas of other patients. In some they are not expected because the sockets have been worn for only a few years-less than the eight years or so required theoretically for the onset of surface fatigue-but in sockets used for longer times there also appears to be no sign of surface fatigue. This might be explained by a level of activity lower than that used to estimate the onset of fatigue in the sockets.

An interesting surface topography has been observed in the high wear areas of a few sockets and can only be described as "parallel ripples" and "bald patches" (Fig. 14). These were first observed in Patient HB7 who was seventy-two years old at the time of operation. Afterwards, she continued to live alone and was most independent and active, even at the age of eighty-two. When she died, her THR had been in use for twelve years.

An unusual feature of the parallel ripples is the fact that the parallelism continued across the entire area of 


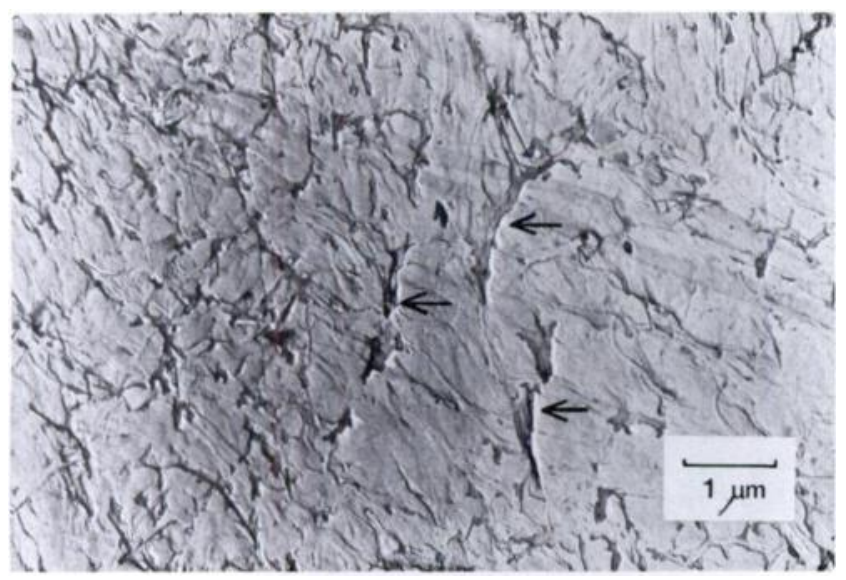

Fig. 13

Surface fatigue cracks (arrowed) and adhesive wear in high wear area in the left acetabular cup of Patient HB3 (Replica 3).

three replicas. This is not consistent with the multidirectional sliding conditions in the hip. The cause of the parallel ripples has not yet been identified. The other notable features, the bald patches, are areas where there are no parallel ripples, only a smooth surface with a few cracks and adhesive pulls (Fig. 14). These may be areas where a piece of polymer has flaked off the surface. This could occur by surface fatigue: fatigue cracks form and propagate tangentially under the surface until a flake of polymer detaches itself or spalls. In support of this spalling hypothesis, Figure 15 shows part of a surface which might be about to spall. At high magnification, fine cracks were observed around the edge of this feature.

Subsequent examination of other sockets has shown that parallel ripples are not a unique feature of Patient HB7. They have been observed on seven sockets: of these, three have exhibited parallel ripples consistently on all replicas from their high wear areas and the other four have patches of parallel ripples in otherwise multi-directional, adhesive wear features. One other patient showed spall features.

When the acetabular cups are arranged chronologically in order of service age, it is noticed that parallel ripples are dominant in those hips which have been used for about eight years or longer (Table I). Thus it is

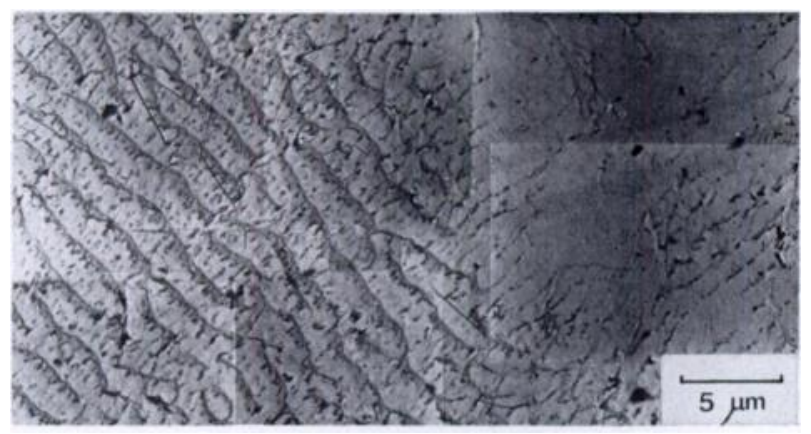

Fig. 14

Parallel ripples and bald patch on the high wear area on the acetabular cup from Patient HB7. possible that, together with the spalling, the parallel ripples may be connected with the onset of surface fatigue. However, why surface fatigue should produce such an oriented topography is hard to explain.

\section{DISCUSSION}

Ultra-high molecular weight polyethylene acetabular cups appear to undergo several processes in the human body during fourteen years of use. Initially, there is a running-in period where surface irregularities are removed abrasively. As the patient regains mobility, the loading geometry of the hip leads to preferential "wear" on the superior half of the cup. This "high wear" area is smooth and polished and adhesive wear features may be seen. The inferior part of the cup (the "low wear" area) is consequently lightly and infrequently loaded and the original machining marks are retained with abrasive wear features from the running-in period. After service times of the order of seven or eight years the "high wear" area is relatively deeply "worn" and the femoral head has effectively created a new socket in this region.

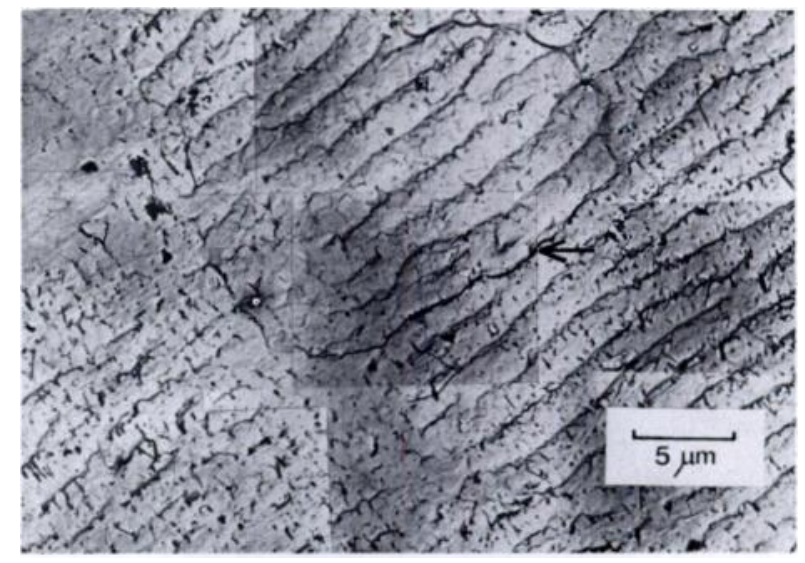

Fig. 15

Parallel ripples and spalling particle (arrowed) on the high wear area on the acetabular cup from Patient HB7.

This results in a ridge between the high and low wear areas. After eight to nine years of use, there is evidence to suggest that, in the "high wear" area, there could be fatigue of the surface of the UHMWPE. This is characterised by the appearance of fine cracks and spall marks in addition to adhesive wear features.

The above explains the appearance of the acetabular cups "worn" in the human body, but does not show why UHMWPE should "wear" faster in the body than is predicted by laboratory tests, where the wear rate for surface fatigue is still less than the clinical rate. However, some of the microscopic features indicate that additional deformation processes occur during the wear of UHMWPE cups in the body.

The presence of joint fluid may lead to stress corrosion (Traveniou 1976). This is more insidious than pure surface fatigue and proceeds more rapidly than 
when either the stress or the fluid acts alone. This phenomenon is well known in metals and similar effects have been observed in polymers, often referred to as environmental cracking. Cracks and spalls may well be the result of stress corrosion. However, because the load on the hip oscillates, the metallurgical term "corrosion fatigue" should perhaps be used. Again, this conjoint action of a fluctuating stress and a fluid on a loaded member can lead to rapid deterioration and weakening of the material. If stress corrosion or corrosion fatigue are taking place, then this would account for the increased clinical "wear" rate.

The hip is at a temperature of 37 degrees Celsius. At this temperature, creep of UHMWPE could be quite significant. Some initial creep tests on acetabular cups at 37 degrees Celsius have been carried out at Leeds University (Smith 1978) which indicate that creep is much more important than wear initially, but that the creep rate decreases exponentially and after long periods of time the creep wear rates are likely to be similar. If creep is occurring, the mechanism is probably one of plastic flow of material away from the highly compressed region (the "high wear" area) down towards the relatively lightly-stressed region (the "low wear" area). The authors propose that the influx of this extra material could be accommodated by microscopic surface buckling and wrinkling in the "low wear" area. Hence, the observed wrinkles and cracks may indicate that such creep is taking place.

Related to the corrosion factor is "three-body abrasive wear". This occurs when abrasive particles get trapped between two rubbing surfaces. The resulting wear rate is higher than that without the particles. If, during fatigue of the cup, polymer spalls or flakes are produced in abundance, these may accumulate in the joint cavity and lead to three-body abrasive wear.

Finally, there is the possibility of fretting fatigue. This is observed in metals when contacting surfaces undergo small, oscillatory, tangential displacements. Fretting fatigue proceeds more rapidly than surface fatigue and is associated with surface cracks and detached particles (Hoeppner and Goss 1974). Probably there is some fretting motion in the hip, especially in elderly patients. If so, fretting fatigue may contribute to the high clinical wear rate.

It is doubtful whether these four mechanisms act in isolation; it is probable that they all occur to some extent in addition to true wear.

\section{REFERENCES}

Atkinson, J. R. (1975) Mechanical properties and wear behaviour of plastics in relation to their use in prostheses. British Polymer Journal, 7, 93-107.

Atkinson, J. R., Brown, K. J., and Dowson, D. (1978) The wear of isotropic polyethylene against dry stainless steel in uni-directional motion. Transactions of the American Society of Mechanical Engineers, Journal of Lubrication Technology, 100, 208-218.

Brown, K. J., Atkinson, J. R., Dowson, D., and Wright, V. (1976) The wear of ultra high molecular weight polyethylene and a preliminary study of its relation to the in vivo behaviour of replacement hip joints. Wear, 40, 255-264.

Charnley, J. (1978) Unpublished data.

Charnley, J., and Cupic, Z. (1973) The nine and ten year results of the low-friction arthroplasty of the hip. Clinical Orthopaedics and Related Research, 95, 9-25.

Dowson, D. (1966-67) Modes of lubrication in human joints. Proceedings of the Institution of Mechanical Engineers, 181 Pt. 3J, $45-54$.

Duff-Barclay, I., and Spillman, D. T. (1966-67) Total human hip joint prostheses-A laboratory study of friction and wear. Proceedings of the Institution of Mechanical Engineers, 181 Pt. 3J, 90-103.

Hoeppner, D. W., and Goss, G. L. (1974) Metallographic analysis of fretting fatigue damage in Ti-6Al-4V MA and 7075-T6 aluminum. Wear, 27, $175-187$.

MacConaill, M. A. (1966-67) Basic anatomy of weight-bearing joints. Proceedings of the Institution of Mechanical Engineers, 181 Pt. 3 J, 1-7.

Paul, J. P. (1976) Force actions transmitted by joints in the human body. Proceedings of the Royal Society of London, B192, 163-172.

Smith, P. R. (1978) Personal communication. Department of Metallurgy. University of Leeds, Leeds.

Traveniou, S. Z. M. (1976) Irregularities in the wear of plastics materials in the hip joint. Plastics and Rubber, 1, $194-196$. 